\title{
Dynamic Spiral CT
}

National Cancer Institute

\section{Source}

National Cancer Institute. Dynamic Spiral CT. NCI Thesaurus. Code C19913.

A type of computed tomography in which the patient is moved through the scanner continuously rather than in increments, so that the path of the beam through the patient is a continuous spiral. 